\title{
Extension of Partitional Clustering Methods for Handling Mixed Data
}

\author{
Yosr Naija \\ LIP2, Faculty of Science of Tunis \\ Campus Universitaire \\ 2092 El-Manar Tunis, Tunisia \\ yosr.naija@gmail.com
}

\author{
Salem Chakhar \\ LAMSADE, University of Paris-Dauphine \\ Place du Maréchal de Lattre de Tassigny \\ 75775 Paris Cedex 16, Paris, France \\ salem.chakhar@dauphine.fr \\ and \\ École Centrale Paris, Grande Voie des Vignes \\ 92295 Châtenay-Malabry Cedex, France \\ salem.chakhar@ecp.fr \\ Riadh Robbana \\ LIP2, Faculty of Science of Tunis \\ Campus Universitaire \\ 2092 El-Manar Tunis, Tunisia \\ riadh.robbana@fst.rnu.tn
}

Kaouther Blibech

LIP2, Faculty of Science of Tunis

Campus Universitaire

2092 El-Manar Tunis, Tunisia

kaouther.blibech@gmail.com

\begin{abstract}
Clustering is an active research topic in data mining and different methods have been proposed in the literature. Most of these methods are based on the use of a distance measure defined either on numerical attributes or on categorical attributes. However, in fields such as road traffic and medicine, datasets are composed of numerical and categorical attributes. Recently, there have been several proposals to develop clustering methods that support mixed attributes. There are three basic categories of clustering methods: partitional methods, hierarchical methods and density-based methods. This paper proposes an extension of partitional clustering methods devoted to mixed attributes. The proposed extension looks to create several partitions by using numerical attributes-based clustering methods and then chooses the one that maximizes a measure-called "homogeneity degree"-of these partitions according to categorical attributes.
\end{abstract}

\section{Introduction}

In applications such as telecommunications, road traffic, medicine and banking there is often a need to extract data measuring the evolution of some parameters that are useful for decision making. These parameters can be numerical (e.g. phone consumption, flow of cars, consumption of to- bacco, balances of the customer) or categorical (e.g. day of the week, antecedents of a disease in a family, unemployed). Decision makers (e.g. traffic experts, doctors, or bank managers) have to understand and analyse these data to make decisions. For example, an expert of traffic should understand why there is a congestion in a period of day to avoid this situation, a doctor needs to know the reasons for which a group of people is ill to better dealt with the problem. They should find the factors that influence a given behavior.

Clustering is a widely used technique in data mining applications to achieve this goal. Generally, decision makers have a limited information about datasets that they hold. Clustering allows to enrich these datasets and to identify similar or different behaviors between them. Concretely, it allows to partition a set of objects into classes based on defined criteria so that objects in the same class are more similar than objects within different classes $[5,9]$.

In the second half of 20th century, data mining community has proposed clustering methods that handle objects described only by numerical attributes such as K-means [12], or only by categorical ones such as K-mode [7]. However, as mentioned above, datasets in real-world applications are composed of both types of attributes. Since the late of 1990th, there have been several proposals to develop clustering methods that support mixed attributes (numerical and categorical ones). Most efforts have been oriented towards the extension of conventional clustering algorithms giving a new distance measure that handles mixed attributes. It 
is important to note that both numerical and categorical attributes need to be considered during classification. However, we think that numerical attributes are more discriminative than categorical ones since they explain better the differences that may exist between objects. Categorical attributes can be used latter to explain some behaviors. The use of weighted-sum like aggregation rules by combining both numerical and categorical attributes may be not suitable since the definition of weights is not an obvious task.

In this paper, we present a clustering approach that deals with mixed attributes while giving more importance to numerical ones. There are three basic categories of clustering methods: partitional methods, hierarchical methods and density-based methods. The paper proposes an extension to partitional clustering methods devoted to mixed attributes. The extension looks to create several partitions by using numerical attributes-based clustering methods and then chooses the one that maximizes a measure, that we called "homogeneity degree", of these partitions according to categorical attributes.

This paper is organized as follows. Section 2 discusses some related work. Section 3 details the proposed approach. Section 4 presents an extended mixed-attributes partitional clustering algorithm. Section 5 presents some numerical results and discusses some important points. Section 6 concludes the paper.

\section{Related work}

\subsection{Clustering and clustering approaches}

Clustering allows to partition a set of objects into classes based on defined criteria so that objects in the same class are more similar than objects within different classes $[5,9]$. More formally, given a set of $n$ objects $X=$ $\left\{O_{1}, O_{2}, \ldots, O_{n}\right\}$, clustering aims to find a function

$$
f: X \rightarrow\left\{C_{1}, C_{2}, \ldots, C_{k}\right\}
$$

that assigns each object $O_{i} \in X$ to a class $C_{j}(j=$ $1, \cdots, k)$ ( $k$ represents the number of classes) .

Three main steps are important to cluster objects [9]: (i) feature selection where characteristics of objects (the attributes that describe objects) are identified; (ii) definition of a proximity measure which is often a distance function that will be used to measure similarity between objects. The choice of a given proximity measure depends largely on the application's domain. An example of distance function is the Euclidean distance; and (iii) creation of classes where a clustering method is first identified and then used to create classes.

The basic categories of clustering methods are [5]:
- Partitional methods: these methods aim to decompose objects into $k$ classes. With this approach the number of classes $k$ is generally a predefined parameter. K-means [12], CLARANS [13] and K-mode [7] are examples of partitional clustering methods.

- Hierarchical methods: given $n$ objects, this category of methods aims to create different levels of partitions (from 1 to $n-1$ levels). The levels are built either by merging at each step the two closest classes until obtaining one class or by splitting, at each step, clusters that contain dispersed objects until obtaining $n$ classes or satisfying a given criterion. Examples are: agglomerative methods, divisive methods [10], BIRCH [15] and CURE [4] methods.

- Density-based methods: these methods group objects into classes so that the surface or volume of these classes are dense "enough". Density is computed using pre-defined thresholds. DBSCAN [3] is one of the most used density-based methods. Squeezer [6] is a density-based method devoted to categorical attributes.

There are other categories of clustering methods such as Grid-based methods but they are used only in specific domains like spatial data mining.

\subsection{Mixed attributes-based clustering}

There are three intuitive ideas to support mixed attributes clustering:

- The conversion of each categorical attribute into a numerical attribute. The clustering methods based on numerical attributes can then be used. However, it is difficult sometimes to code a categorical attribute without understanding the meaning of this attribute [1]. For example, the conversion of an attribute color is more difficult than the conversion of an attribute month.

- The discretization of numerical attributes so that each numerical attribute is converted into a categorical one by using a discretization method. Then the objects can be clustered by using one of the clustering methods based on categorical attributes. DSqueezer (Discretizing before Using Squeezer) [6] method follows this idea. The principal limit of this type of approach is the loss of information generated by discretizing.

- Conversion of the categorical attributes into binary ones: each categorical attribute $A_{i}$ with $d$ distinct values is converted into $d$ binary attributes where each attribute takes value 1 if the object is described by this attribute, 0 if not. In [14], Ralambondrainy follows this principle to create classes of objects with mixed 
attributes. The disadvantage of this approach is that the conversion increases the number of attributes describing the objects. Moreover, with this approach attributes are less meaningful after conversion [2].

More recently, more emphasized methods allowing to cluster objects described by mixed attributes have been proposed in the literature. These methods represent extensions of conventional methods (numerical attributes-based clustering methods or categorical attributes-based clustering methods). Extensions consist in using a measure distance that supports mixed attributes. The three clustering steps mentioned earlier still apply with these extended methods.

$\mathrm{K}$-prototype [8] is one of the first extended methods proposed in the literature. It is a combination of K-means [12] and K-mode [7] methods. The distance measure used with $\mathrm{K}$-prototype is the sum of Euclidean distance, which is used to compute the distance between numerical attributes, and the simple matching measure used to dealt with categorical attributes where the distance between two objects is computed as the number of different attributes values between these objects. To build classes, K-prototype follows the same steps as K-means and K-mode.

The authors of [2] propose a clustering method based on the algorithm of agglomerative hierarchical clustering methods and on a distance hierarchy that expresses similarity between attributes' values. Every attribute is represented by a hierarchy. For categorical attributes this hierarchy is a tree where each node represents a possible value of the attribute and every link is valued by a weight representing the distance between these values. Concerning numerical attributes, the hierarchy is represented by only two nodes corresponding to the minimal and maximal values of the attribute. The distance between two objects is the total of distances between values of objects' attributes based on the hierarchy distance. Given this distance measure, the [2]'s method begins by representing each class by one object and then an additional measure is used iteratively to merge the closest classes.

UsmSquezer (Squeezer for Unified Similarity Measures) [6] is an extension of the categorical attribute-based Squeezer method. The authors propose a similarity measure between an object $O$ and a class $C$, which is the sum of similarity measure between categorical attributes and similarity measure between numerical attributes. The first one computed on basis of the proportion of objects of $C$ having the same values as the studied object $O$. The second one is computed on the basis of the difference between values of each numerical attribute $A$ of $O$ and the mean value of the studied attribute for all objects of the class. The clustering algorithm of UsmSquezer remains the same as Squeezer.

There are several other methods supporting mixed attributes $[1,11]$. Most of them rely on the definition of a new distance measure that combines numerical and categorical attributes. However and as mentioned previously in Section 1, we think that numerical attributes are more discriminative than categorical. The approach that we propose in the next section is an extension to partitional clustering methods.It looks to create several partitions by using numerical attribute-based clustering methods and then chooses the one that maximizes the "homogeneity degree" of these partitions according to categorical attributes.

\section{Proposed homogeneity degree-based cluster- ing approach}

The basic idea of the proposed approach is to apply a numerical attributes-based clustering method several times on the same datasets while changing some initial parameters such as the number of classes or the similarity thresholds. Then, for every obtained partition, a measure called homogeneity degree is computed. The homogeneity degree measures the homogeneity of a partition according to categorical attributes. Finally, the partition that maximizes the homogeneity degree is selected. Algorithm 1 in Section 4 illustrates this idea. The objective of this section is to show how homogeneity degree is computed

The homogeneity degree of a partition depends on the homogeneity degrees of its classes and the homogeneity degrees of attributes domains. Some useful definitions are introduced first.

\subsection{Basic definitions}

Definition 3.1 (Domain of categorical attribute). The domain of a categorical attribute $A_{i}$ is denoted $\operatorname{DOM}\left(A_{i}\right)=$ $\left\{a_{i 1}, a_{i 2}, \ldots, a_{i t}\right\}$ where $a_{i j}$ is a possible value of $A_{i}$ and $t$ is the number of possible values.

Definition 3.2 (Hierarchy of categorical attributes). A hierarchy organizes attributes according to a "finer than" relationship, along with their level of details. Let $A_{1}$ and $A_{2}$ be two categorical attributes. Then, $A_{1} \subseteq_{H A} A_{2}$ signifies that $A_{1}$ is less general (or more finer) than $A_{2}$. If there is not any hierarchical relation between $A_{1}$ and $A_{2}$, $A_{1}$ and $A_{2}$ are said to be non hierarchical. This is denoted by $A_{1} \varsubsetneqq \mathrm{HA} A_{2}$.

Example 1. Let month and trimester be two categorical attributes. Let DOM(month $)=\{1,2, \ldots, 12\}$ and $D O M($ trimester $)=\{1,2,3,4\}$. Then, month $\subseteq_{H A}$ trimester.

Definition 3.3 (Labels of an object). A label is a set of values of categorical attributes with no hierarchical relationship among them. Let $A_{1}, A_{2}, \ldots, A_{n}$ be $n$ categorical attributes. The set of labels $L$ associated with attributes 
$A_{1}, A_{2}, \ldots, A_{n}$ is defined as the Cartesian product of the domains of $A_{1}, A_{2}, \ldots, A_{n}: \operatorname{DOM}\left(A_{1}\right) \times \operatorname{DOM}\left(A_{2}\right) \times$ $\cdots \times \operatorname{DOM}\left(A_{n}\right)$. Accordingly, a label $l_{i} \in L$ is an instance of $\operatorname{DOM}\left(A_{1}\right) \times \operatorname{DOM}\left(A_{2}\right) \times \cdots \times \operatorname{DOM}\left(A_{n}\right)$. The notation Label $(O)=l_{i}$ means that object $O$ is described by label $l_{i}$.

Example 2. Let $A_{1}, A_{2}$ and $A_{3}$ be three categorical attributes associated respectively with the day number of the week (from 1 to 7 ), the type of day ( $Y$ if it is a holiday and $N$ if not) and the month number (from 1 to 12). Figure 1 provides the set L of labels. The label $l_{12}$ (1 Y 12) for instance corresponds to a Monday of December, which is also a holiday.

$\mathrm{l}_{1}: 1 \mathrm{Y} 1$
$\mathrm{l}_{2}: 1 \mathrm{Y} 2$
$\ldots$
$\mathrm{l}_{12}: 1 \mathrm{Y} 12$
$\mathrm{l}_{13}: 1 \mathrm{~N} 1$
$\mathrm{l}_{14}: 1 \mathrm{~N} 2$
$\ldots$
$\mathrm{l}_{24}: 1 \mathrm{~N} 12$
$\mathrm{l}_{25}: 2 \mathrm{Y} 1$
$\mathrm{l}_{26}: 2 \mathrm{Y} 2$
$\ldots$
$\ldots$
$\ldots$
$\mathrm{l}_{167}: 7 \mathrm{~N} 11$
$\mathrm{l}_{168}: 7 \mathrm{~N} 12$

\section{Figure 1. Example of labels}

\subsection{Class homogeneity according to cate- gorical attributes}

Naturally, a class is homogeneous according to categorical attributes if its objects have the same label(s). However, a crisp definition of the notion of homogeneity may be very restrictive in practice and makes homogeneity largely depending on the application's domain and on the datasets. The homogeneity degree concept as introduced in the rest of this section provides a fuzzy measure of homogeneity. Indeed, homogeneity degree takes its values in the range $[0,1]$, where 1 indicates that the class is fully homogeneous and 0 indicates that the class is not homogeneous. A value between 0 and 1 represents the level to which a class is homogeneous.

The definition of homogeneity degree requires the use of two thresholds $\alpha \in[0,1]$ and $\beta \in[0,1]$ assuring the co- herence of results. The practical utility of these parameters will be better explained in 5.3.1.

Next, some useful notations are introduced.

- $X=\left\{O_{1}, O_{2}, \ldots, O_{n}\right\}$ : a set of $n$ objects.

- $L=\left\{l_{1}, l_{2}, \ldots, l_{m}\right\}$ : a set of $m$ labels $(m \leq n)$.

- $P_{k}\left(C_{1}, C_{2}, \ldots, C_{k}\right)$ : a partition of $k$ classes.

- $S C_{i j}=\left\{O_{r} \in C_{i}: \operatorname{Label}\left(O_{r}\right)=l_{j}\right\}$ : the set of objects of class $C_{i}$ having the label $l_{j}$.

- $S_{j}=\left\{O_{r} \in X: \operatorname{Label}\left(O_{r}\right)=l_{j}\right\}$ : the set of objects of $X$ having the label $l_{j}$.

- $\|\cdot\|$ : set cardinality symbol.

\subsubsection{Membership degree of a label in re- spect to a domain}

The membership degree $M D_{\alpha}\left(l_{j}, C_{i}\right)$ of a label $l_{j}$ in a class $C_{i}$ in respect to a domain expresses the proportion of objects of $C_{i}$ described by label $l_{j}$ in respect to the total number of objects of $X$ having the label $l_{j} . M D_{\alpha}\left(l_{j}, C_{i}\right)$ is defined as follows:

$M D_{\alpha}\left(l_{j}, C_{i}\right)= \begin{cases}\frac{\left\|S C_{i j}\right\|}{\left\|S_{j}\right\|}, & \text { if } \frac{\left\|S C_{i j}\right\|}{\left\|S_{j}\right\|} \geq \alpha \text { and }\left\|S_{j}\right\| \neq 0 \\ 0, & \text { Otherwise }\end{cases}$

As it is shown in Eq. $1, M D_{\alpha}\left(l_{j}, C_{i}\right)$ will be equal to 0 any time the proportion of objects described by label $l_{j}$ is strictly less than $\alpha$. This ensures that $C_{i}$ contains at least $\alpha \%$ of objects of $X$.

\subsubsection{Homogeneity degree in respect to a domain}

The homogeneity degree of a class $C_{i}$ in respect to a domain, denoted by $H D_{\alpha}\left(C_{i}\right)$, is defined through Eq. 2:

$$
H D_{\alpha}\left(C_{i}\right)=\left\{\begin{array}{l}
\frac{1}{B} \cdot \sum_{j=1}^{m} M D_{\alpha}\left(l_{j}, C_{i}\right), \text { if } B \neq 0 \\
0, \text { Otherwise }
\end{array}\right.
$$

where $B=\left\|\left\{l_{k}: l_{k} \in L \wedge M D_{\alpha}\left(l_{k}, C_{i}\right)>0\right\}\right\|$, that is, the number of labels $l_{k}$ such that $M D_{\alpha}\left(l_{k}, C_{i}\right)$ is strictly positive.

$H D_{\alpha}\left(C_{i}\right)$ represents the average of labels' membership degrees. Other formula such as the product of $M D_{\alpha}\left(l_{k}, C_{i}\right)$ $(k=1, \cdots, m)$ or the maximum or minimum value of $M D_{\alpha}\left(l_{k}, C_{i}\right)(k=1, \cdots, m)$ may also apply here. 


\subsubsection{Membership degree of a label in re- spect to a class}

The membership degree $M C_{\alpha}\left(l_{j}, C_{i}\right)$ of a label $l_{j}$ in respect to a class $C_{i}$ reflects the proportion of objects of $C_{i}$ described by $l_{j}$. In other words, $M C_{\alpha}\left(l_{j}, C_{i}\right)$ permits to measure the importance of label $l_{j}$ to class $C_{j}$. Formally, $M C_{\alpha}\left(l_{j}, C_{i}\right)$ is computed as follows:

$$
M C_{\alpha}\left(l_{j}, C_{i}\right)= \begin{cases}\frac{\left\|S C_{i j}\right\|}{\left\|C_{i}\right\|}, & \text { if } M D_{\alpha}\left(l_{j}, C_{i}\right) \geq \alpha \\ 0, & \text { Otherwise }\end{cases}
$$

As it is shown in Eq. 3, only labels for which $M D_{\alpha} \geq \alpha$ are included in the definition of $M C_{\alpha}\left(l_{j}, C_{i}\right)$. This ensures that only the most important labels are considered in the computing of $M C_{\alpha}\left(l_{j}, C_{i}\right)$.

\subsubsection{Partial homogeneity degree of a class}

The partial homogeneity degree $H C_{\alpha}\left(C_{i}\right)$ of class $C_{i}$ is given by Eq. 4:

$$
H C_{\alpha, \beta}\left(C_{i}\right)=\left\{\begin{array}{l}
\sum_{j=1}^{m} M C_{\alpha}\left(l_{j}, C_{i}\right) \\
\text { if } \sum_{j=1}^{m} M C_{\alpha}\left(l_{j}, C_{i}\right) \geq \beta \\
0, \\
\text { Otherwise }
\end{array}\right.
$$

The threshold $\beta$ used in the definition of $H C_{\alpha}\left(C_{i}\right)$ ensures that only important labels are considered for computing the partial homogeneity degree of the class.

\subsubsection{Overall homogeneity degree of a class}

The overall homogeneity degree of a class $C_{i}$ takes into account the homogeneity degree of $C_{i}$ in respect to the domain and the partial homogeneity of $C_{i}$. It is denoted by $D_{\alpha, \beta}\left(C_{i}\right)$ and computed through Eq. 5 hereafter:

$$
D_{\alpha, \beta}\left(C_{i}\right)=H D_{\alpha}\left(C_{i}\right) \cdot H C_{\alpha, \beta}\left(C_{i}\right)
$$

Intuitively, a class $C_{i}$ is more homogeneous (resp. less homogeneous) than a class $C_{j}$ if and only if $D_{\alpha, \beta}\left(C_{i}\right)>$ $D_{\alpha, \beta}\left(C_{j}\right)\left(\right.$ resp. $D_{\alpha, \beta}\left(C_{i}\right)<D_{\alpha, \beta}\left(C_{j}\right)$ ).

\subsection{Partition homogeneity degree accord- ing to categorical attributes}

Let $P_{k}\left(C_{1}, C_{2}, \ldots, C_{k}\right)$ be a partition of $k$ classes. The homogeneity degree $D P_{\alpha, \beta}\left(P_{k}\right)$ of $P_{k}$ is defined by the Eq. 6 below:

$$
D P_{\alpha, \beta}\left(P_{k}\right)=\frac{1}{k} \cdot \sum_{i=1}^{k} D_{\alpha, \beta}\left(C_{i}\right)
$$

It is important to note that other formula may also apply such as:

$$
\begin{gathered}
D P_{\alpha, \beta}(P)=\prod_{i=1}^{i=k} D_{\alpha, \beta}\left(C_{i}\right), \\
D P_{\alpha, \beta}(P)=\min \left(D_{\alpha, \beta}\left(C_{i}\right), \cdots, D_{\alpha, \beta}\left(C_{k}\right)\right),
\end{gathered}
$$

or

$$
D P_{\alpha, \beta}(P)=\max \left(D_{\alpha, \beta}\left(C_{i}\right), \cdots, D_{\alpha, \beta}\left(C_{k}\right)\right) .
$$

In the rest of this paper we suppose that Eq. 6 is used.

The value of $D P_{\alpha, \beta}\left(P_{k}\right)$ varies between 0 and 1 . If $D P_{\alpha, \beta}\left(P_{k}\right)=1$, the partition is said to be fully homogeneous and if $D P_{\alpha, \beta}\left(P_{k}\right)=0$, then the partition is non homogeneous. Otherwise, the partition $P_{k}$ is homogenous to a level equal to $D P_{\alpha, \beta}\left(P_{k}\right)$.

\subsection{Illustrative example}

Consider a set $X$ of objects described by two attributes:

- Attribute $A_{1}$ indicates if a day is a working day (between Monday and Friday) or not: $\operatorname{DOM}\left(A_{1}\right)=$ $\{Y, N\}$.

- Attribute $A_{2}$ indicates if a day is a holiday or not: $\operatorname{DOM}\left(A_{2}\right)=\{Y, N\}$.

The $L=\left\{l_{1}=Y Y, l_{2}=Y N, l_{3}=N Y, l_{4}=N N\right\}$ be the set of labels. Suppose that $\left\|S_{1}\right\|=5,\left\|S_{2}\right\|=10$, $\left\|S_{3}\right\|=6$, and $\left\|S_{4}\right\|=4$. Let $C_{1}$ and $C_{2}$ be two classes defined on $X$. The content of these two classes is shown in Figure 2. Class $C_{1}$ contains 13 objects (two are described by label $l_{1}$, nine by $l_{2}$ and two by $l_{4}$ ). Class $C_{2}$ contains 12 objects (three are described by label $l_{1}$, one by $l_{2}$, six by $l_{3}$ and two by $l_{4}$ ).

Consider now class $C_{1}$ and suppose that $\alpha=0.5$ and $\beta=0.1$. Using Eq. 1 and Eq. 3, we get:

- $M D_{0.5}\left(l_{1}, C_{1}\right)=0 ; M C_{0.5}\left(l_{1}, C_{1}\right)=0$,

- $M D_{0.5}\left(l_{2}, C_{1}\right)=0.9 ; M C_{0.5}\left(l_{2}, C_{1}\right)=0.64$,

- $M D_{0.5}\left(l_{3}, C_{1}\right)=0 ; M C_{0.5}\left(l_{3}, C_{1}\right)=0$,

- $M D_{0.5}\left(l_{4}, C_{1}\right)=0.5 ; M C_{0.5}\left(l_{4}, C_{1}\right)=0.22$. 


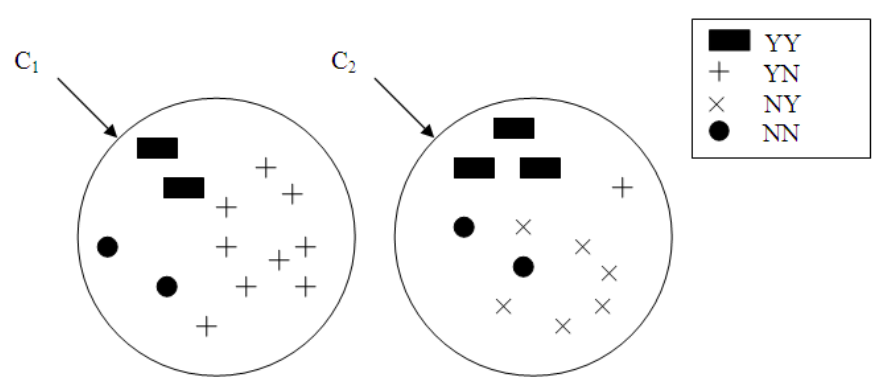

Figure 2. Classes $C_{1}$ and $C_{2}$

For instance: $M D_{0.5}\left(l_{1}, C_{1}\right)=0$ (since $\frac{\left\|S C_{1,1}\right\|}{\left\|S_{1}\right\|}=\frac{2}{5}=$ $0.4<\alpha)$ and $M D_{0.5}\left(l_{4}, C_{1}\right)=\frac{\left\|S C_{4,1}\right\|}{\left\|S_{4}\right\|}=\frac{2}{4}=0.5$.

Then, by using Eq. 2 and Eq. 4, we get:

- $H D_{0.5}\left(C_{1}\right)=\frac{1}{2}\left(M D_{0.5}\left(l_{2}, C_{1}\right)+M D_{0.5}\left(l_{4}, C_{1}\right)\right)=$ 0.7 ,

- $H C_{0.5,0.1}\left(C_{1}\right)=M C_{0.5}\left(l_{1}, C_{1}\right)+M C_{0.5}\left(l_{2}, C_{1}\right)+$ $M C_{0.5}\left(l_{3}, C_{1}\right)+M C_{0.5}\left(l_{4}, C_{1}\right)=0.86$.

Next, the homogeneity degrees of classes $C_{1}$ and $C_{2}$ can be computed by Eq. 5. This leads to:

$$
\begin{aligned}
D_{0.5,0.1}\left(C_{1}\right) & =H D_{0.5}\left(C_{1}\right) \cdot H C_{0.5,0.1}\left(C_{1}\right) \\
& =0.7 \cdot 0.86 \\
& =0.6 \\
& \\
D_{0.5,0.1}\left(C_{2}\right) & =H D_{0.5}\left(C_{2}\right) \cdot H C_{0.5,0.1}\left(C_{2}\right) \\
& =0.64
\end{aligned}
$$

Finally, the homogeneity degree of partition $P_{2}\left(C_{1}, C_{2}\right)$ is computed by Eq. 6:

$$
\begin{aligned}
D P_{0.5,0.1}\left(P_{2}\right) & =\frac{1}{2}\left(D_{0.5,0.1}\left(C_{1}\right)+D_{0.5,0.1}\left(C_{2}\right)\right) \\
& =\frac{1}{2}(0.6+0.64) \\
& =0.62
\end{aligned}
$$

\begin{tabular}{|c|c|c|c|c|}
\hline$\alpha$ & $\beta$ & $H D_{\alpha}\left(C_{1}\right)$ & $H C_{\alpha, \beta}\left(C_{1}\right)$ & $D_{\alpha, \beta}\left(C_{1}\right)$ \\
\hline \multirow{3}{*}{0.1} & 0.1 & \multirow{3}{*}{0.6} & 1 & 0.6 \\
\hline & 0.5 & & 1 & 0.6 \\
\hline & 0.9 & & 1 & 0.6 \\
\hline \multirow{3}{*}{0.5} & 0.1 & \multirow{3}{*}{0.7} & 0.86 & 0.6 \\
\hline & 0.5 & & 0.86 & 0.6 \\
\hline & 0.9 & & 0 & 0 \\
\hline \multirow{3}{*}{0.9} & 0.1 & \multirow{3}{*}{0.9} & 0.64 & 0.58 \\
\hline & 0.5 & & 0.64 & 0.58 \\
\hline & 0.9 & & 0 & 0 \\
\hline \multirow{3}{*}{1} & 0.1 & \multirow{3}{*}{0} & 0 & 0 \\
\hline & 0.5 & & 0 & 0 \\
\hline & 0.9 & & 0 & 0 \\
\hline
\end{tabular}

Tables 1, 2 and 3 hereafter give the values of $D_{\alpha, \beta}\left(C_{1}\right)$,

\begin{tabular}{|c|c|c|c|c|}
\hline$\alpha$ & $\beta$ & $H D_{\alpha}\left(C_{2}\right)$ & $H C_{\alpha, \beta}\left(C_{2}\right)$ & $D_{\alpha, \beta}\left(C_{2}\right)$ \\
\hline \multirow{3}{*}{0.1} & 0.1 & \multirow{3}{*}{0.55} & 1 & 0.55 \\
\hline & 0.5 & & 1 & 0.55 \\
\hline & 0.9 & & 1 & 0.55 \\
\hline \multirow{3}{*}{0.5} & 0.1 & \multirow{3}{*}{0.7} & 0.91 & 0.64 \\
\hline & 0.5 & & 0.91 & 0.64 \\
\hline & 0.9 & & 0.91 & 0.64 \\
\hline \multirow{3}{*}{0.9} & 0.1 & \multirow{3}{*}{1} & 0.36 & 0.36 \\
\hline & 0.5 & & 0 & 0 \\
\hline & 0.9 & & 0 & 0 \\
\hline \multirow{3}{*}{0.9} & 0.1 & \multirow{3}{*}{1} & 0.36 & 0.36 \\
\hline & 0.5 & & 0 & 0 \\
\hline & 0.9 & & 0 & 0 \\
\hline
\end{tabular}
$D_{\alpha, \beta}\left(C_{2}\right)$ and $D P_{\alpha, \beta}$ for some values of $\alpha$ and $\beta$. It is easy to see that for $\alpha=0.1$ and $\beta=0.5$, class $C_{1}$ is more homogeneous than class $C_{2}$, while for $\alpha=0.5$ and $\beta=$ $0.1, C_{2}$ is more homogeneous than $C_{1}$.
Table 1. Values of $H D_{\alpha}\left(C_{1}\right), H C_{\alpha}\left(C_{1}\right)$ and $D_{\alpha, \beta}\left(C_{1}\right)$

Table 2. Values of $H D_{\alpha}\left(C_{2}\right), H C_{\alpha}\left(C_{2}\right)$ and $D_{\alpha, \beta}\left(C_{2}\right)$

\begin{tabular}{|c|c|c|c|c|}
\hline$\alpha$ & $\beta$ & $D_{\alpha, \beta}\left(C_{1}\right)$ & $D_{\alpha, \beta}\left(C_{2}\right)$ & $D P_{\alpha, \beta}\left(P_{2}\right)$ \\
\hline \multirow{3}{*}{0.1} & 0.1 & 0.6 & 0.55 & 0.58 \\
\cline { 2 - 5 } & 0.5 & 0.6 & 0.55 & 0.58 \\
\cline { 2 - 5 } & 0.9 & 0.6 & 0.55 & 0.58 \\
\hline \multirow{3}{*}{0.5} & 0.1 & 0.6 & 0.64 & 0.62 \\
\cline { 2 - 5 } & 0.5 & 0.6 & 0.64 & 0.62 \\
\cline { 2 - 5 } & 0.9 & 0 & 0.64 & 0 \\
\hline \multirow{4}{*}{0.9} & 0.1 & 0.58 & 0.36 & 0.47 \\
\cline { 2 - 5 } & 0.5 & 0.58 & 0 & 0 \\
\cline { 2 - 5 } & 0.9 & 0 & 0 & 0 \\
\hline \multirow{3}{*}{1} & 0.1 & 0 & 0.36 & 0 \\
\cline { 2 - 5 } & 0.5 & 0 & 0 & 0 \\
\cline { 2 - 5 } & 0.9 & 0 & 0 & 0 \\
\hline
\end{tabular}

Table 3. Values of $D P_{\alpha, \beta}\left(P_{2}\right)$ for different values of $\alpha$ and $\beta$

\subsection{Propositions}

This section presents a series of propositions concerning the relationship between $H D_{\alpha}, H C_{\alpha, \beta}, M D_{\alpha}$ and $M C_{\alpha}$.

Proposition 1 (Relation between $H D_{\alpha}$ and $\alpha$ ). Giving $a$ class $C_{i}$,

- If $\left.\max _{j} \frac{\left\|S C_{i j}\right\|}{\left\|S_{j}\right\|}\right)<\alpha$, then $H D_{\alpha}\left(C_{i}\right)=0$ (part 1)

- If $\alpha=1$ and $\nexists l_{j}$ such that $\frac{\left\|S C_{i j}\right\|}{\left\|S_{j}\right\|}=1$, then, $H D_{\alpha}\left(C_{i}\right)=0$ (sub-part 2.1). Otherwise $H D_{\alpha}\left(C_{i}\right)=$ 1 (sub-part 2.2) 
- If there is at least one label $l_{j}$ such that $\frac{\left\|S C_{i j}\right\|}{\left\|S_{j}\right\|}=1$, then $\lim _{\alpha \rightarrow 1}\left(H D_{\alpha}\left(C_{i}\right)\right)=1$ (part 3)

Proof (part 1). Given a class $C_{i}$, if for every label $l_{j}$ $(j=1, \ldots, m), \frac{\left\|S C_{i j}\right\|}{\left\|S_{j}\right\|}<\alpha$, then for every label $l_{j}(j=$ $1, \ldots, m), M D_{\alpha}\left(l_{j}, C_{i}\right)=0$. Then, according to Eq. 2, $H D_{\alpha}\left(C_{i}\right)=0$.

Proof (part 2). The proof of the sub-part 2.1 is trivial because this sub-part is the consequence of part 1. For subpart 2.2, given a class $C_{i}, H D_{\alpha}\left(C_{i}\right)$ is equal to the mean of the $M D_{\alpha}\left(l_{j}, C_{i}\right), \forall l_{j}$, that are different from 0 . When $\alpha=1$, we take into account only the values of $\frac{\left\|S C_{i j}\right\|}{\left\|S_{j}\right\|}$, $\forall l_{j}$, that are greater than 1 to compute $H D_{\alpha}\left(C_{i}\right)$. Then, if there is at least one label $l_{j}$ such that $\frac{\left\|S C_{i j}\right\|}{\left\|S_{j}\right\|}=1$, then $H D_{\alpha}\left(C_{i}\right)=1$

Proof (part 3). As mentioned above, $H D_{\alpha}\left(C_{i}\right)$ is equal to the mean of the $M D_{\alpha}\left(l_{j}, C_{i}\right)$ that are different from 0 . If $\alpha$ is close to 1 , so for the computation of $H D_{\alpha}\left(C_{i}\right)$, we take into account only the values of $\frac{\left\|S C_{i j}\right\|}{\left\|S_{j}\right\|}, \forall l_{j}$, that are greater than $\alpha$. Then, the mean will be greater than $\alpha$.

Proposition 2. Let $\mathrm{Pl}_{j}$ be a set of $r$ classes containing objects having the label $l_{j}$. The following holds:

- $\sum_{i=1}^{r} M D_{\alpha}\left(l_{j}, C_{i}\right)=1$ if $\forall C_{i}, M D_{\alpha}\left(l_{j}, C_{i}\right) \neq 0$ (part 1)

- If for a given class $C_{i} \in P L_{j}, M D_{\alpha}\left(l_{j}, C_{i}\right)>0.5$, then $\forall C_{m} \neq C_{i}$ and $\in P L_{j}, M D_{\alpha}\left(l_{j}, C_{m}\right)<0.5$ (part 2)

Proof (part 1). According to section 3.2.1, $M D_{\alpha}\left(l_{j}, C_{i}\right)$ is equal to the proportion of objects of a class $C_{i}$ described by a label $l_{j}$ in respect to the total number of the objects of $X$ having the label $l_{j}$ when this proportion is greater than $\alpha$. Next, if $\forall C_{i}$, $M D_{\alpha}\left(l_{j}, C_{i}\right) \neq 0$, Then:

$$
\begin{aligned}
\sum_{i=1}^{r} M D_{\alpha}\left(l_{j}, C_{i}\right) & =\frac{\left\|S C_{1 j}\right\|}{\left\|S_{j}\right\|}+\frac{\left\|S C_{2 j}\right\|}{\left\|S_{j}\right\|}+\cdots+\frac{\left\|S C_{r j}\right\|}{\left\|S_{j}\right\|} \\
& =\frac{\left\|S C_{1 j}\right\|+\left\|S C_{2 j}\right\|+\cdots+\left\|S C_{r j}\right\|}{\left\|S_{j}\right\|} \\
& =1
\end{aligned}
$$

Otherwise, we will have some proportions that will not be taken into account.

Proof (part 2). Trivial (consequence of part 1)

Proposition 3 (Relation between $H C_{\alpha, \beta}\left(l_{j}, C_{i}\right)$ and $\left.M D_{\alpha}\left(l_{j}, C_{i}\right)\right)$. Let $C_{i}$ be a class with labels list Labels $\left(C_{i}\right)$. If $\forall l_{j} \in$ Labels $\left(C_{i}\right) M D_{\alpha}\left(l_{j}, C_{i}\right) \geq \alpha$, then $H C_{\alpha, \beta}\left(C_{i}\right)=1$.
Proof. $M D_{\alpha}\left(l_{j}, C_{i}\right) \geq \alpha$ means that $M C_{\alpha}\left(l_{j}, C_{i}\right)=$ $\frac{\left\|S C_{i j}\right\|}{\left\|C_{i}\right\|}$. Consequently, if $\forall l_{j} \in$ Labels $\left(C_{i}\right)$, then $M D_{\alpha}\left(l_{j}, C_{i}\right) \geq \alpha$. Then,

$$
\begin{aligned}
H C_{\alpha, \beta}\left(C_{i}\right) & =\frac{\left\|S C_{i 1}\right\|}{\left\|C_{i}\right\|}+\frac{\left\|S C_{i 2}\right\|}{\left\|C_{i}\right\|}+\cdots+\frac{\left\|S C_{i s}\right\|}{\left\|C_{i}\right\|} \\
& =\frac{\left\|S C_{i 1}\right\|+\left\|S C_{i 2}\right\|+\cdots+\left\|S C_{i j}\right\|}{\left\|C_{i}\right\|} \\
& =1
\end{aligned}
$$

Proposition 4 (Relation between $H C_{\alpha, \beta}$ and $H D_{\alpha}$ ). If $H D_{\alpha}\left(C_{i}\right)=0$, then $H C_{\alpha, \beta}\left(C_{i}\right)=0$.

Proof. $H D_{\alpha}\left(C_{i}\right)=0$ means that $\forall l_{j}, M D_{\alpha}\left(l_{j}, C_{i}\right)>$ 0 . Thus, according to Eq. $3, M C_{\alpha}\left(l_{j}, C_{i}\right)=0, \forall l_{j}$. Consequently, $H D_{\alpha, \beta}\left(C_{i}\right)=0$.

\section{Mixed-attributes clustering algorithm}

Partitional clustering methods permit to obtain $k$ classes of objects. The number of classes $k$ is generally fixed before running the algorithm. The idea of the proposed mixedattributes clustering algorithm is to run several times one of the partitional clustering methods by varying the number of classes $k$ from 2 to a maximal number of classes $k_{\max }$ corresponding to the number of labels. At each iteration we compute the overall homogeneity degree $D P_{\alpha, \beta}$ for different values of $\alpha$ and $\beta$. The values of $\alpha$ and $\beta$ that maximize $D P_{\alpha, \beta}$ are stored. Then, the partition (and so the number of classes) that maximizes $D P_{\alpha, \beta}$ is picked out.

Algorithm 1 that follows implements this idea. The procedure PARTITIONING used in Algorithm 1 may ba any of the conventional partitional clustering methods that permits to obtain a partition of $k$ classes based on numerical attributes.

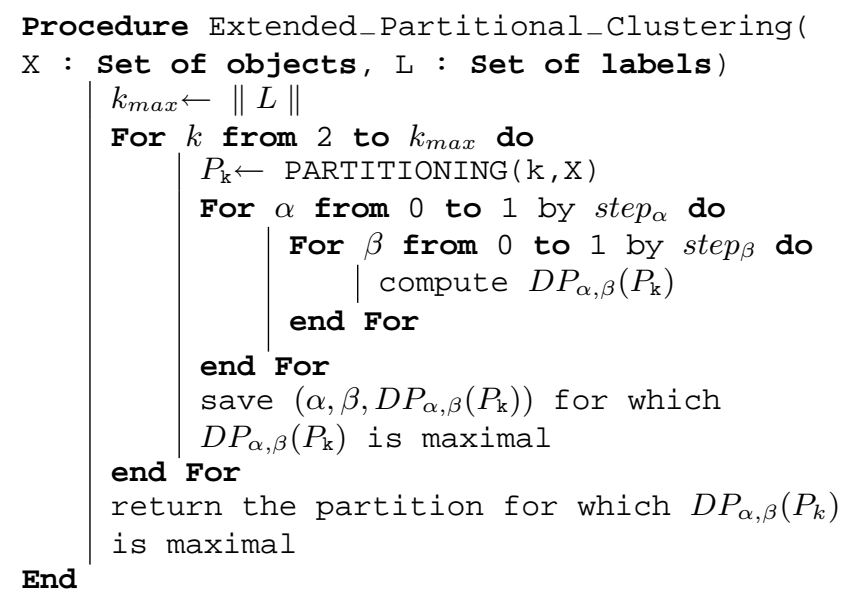

Algorithm 1: Extended partitional clustering algorithm 
Thresholds $\alpha$ and $\beta$ vary from 0 to 1 by step step $_{\alpha}$ and $s t e p_{\beta}$, respectively. The parameters $s t e p_{\alpha}$ and $s t e p_{\beta}$ are chosen by the user before running the algorithm. Both step $p_{\alpha}$ and $s t e p_{\beta}$ must be in the range ]0, 1[. Possible values are: $0.01,0.1$, and 0.2 .

The complexity of the algorithm is $O\left(k_{\max }\right.$. $\left.\left(C P+\frac{1}{\text { step }_{\alpha}} \frac{1}{\operatorname{setp}_{\beta}}\right)\right)$ where $C P$ is the complexity of PARTITIONING algorithm.

\section{Experiment and discussion}

This section presents an application of the proposed algorithm. The well-known K-means [12] partitional clustering algorithm is used.

\subsection{Datasets}

The datasets is composed of 230 objects aleatory generated. Each of these objects is described by 100 numerical attributes. Attributes values used are summed up as follows:

- $E_{1}=\left\{O_{1}, O_{2}, \ldots, O_{50}\right\}: 50$ objects where attributes values vary between 0 and 20 .

- $E_{2}=\left\{O_{51}, O_{52}, \ldots, O_{90}\right\}: 40$ objects where attributes values vary between 150 and 170 .

- $E_{3}=\left\{O_{91}, O_{92}, \ldots, O_{130}\right\}: 40$ objects where attributes values vary between 300 and 320 .

- $E_{4}=\left\{O_{131}, O_{132}, \ldots, O_{200}\right\}: 70$ objects attributes values vary between 450 and 470 .

- $E_{5}=\left\{O_{201}, O_{202}, \ldots, O_{230}\right\}: 30$ objects attributes values vary between 600 and 620 .

For simplicity, the variation range for attributes values is the same for all attributes. This restriction has no consequence on the results.

A set of 10 labels $L=\left\{l_{1}, l_{2}, \ldots, l_{10}\right\}$ is used. These labels are supposed to be created from categorical attributes. The distribution of labels in the different sets is given in Table 4.

\begin{tabular}{|c|c|l|l|l|l|l|}
\hline Label & Number of objects & $E_{1}$ & $E_{2}$ & $E_{3}$ & $E_{4}$ & $E_{5}$ \\
\hline$l_{1}$ & 30 & 18 & 12 & & & \\
\hline$l_{2}$ & 5 & & & 5 & & \\
\hline$l_{3}$ & 25 & 20 & & & 5 & \\
\hline$l_{4}$ & 15 & & & 14 & 1 & \\
\hline$l_{5}$ & 4 & 4 & & & & \\
\hline$l_{6}$ & 21 & & & & 21 & \\
\hline$l_{7}$ & 19 & 2 & & 17 & & \\
\hline$l_{8}$ & 51 & 6 & & 4 & 41 & \\
\hline$l_{9}$ & 30 & & 28 & & 2 & \\
\hline$l_{10}$ & 30 & & & & & 30 \\
\hline Total & $\mathbf{2 3 0}$ & $\mathbf{5 0}$ & $\mathbf{4 0}$ & $\mathbf{4 0}$ & $\mathbf{7 0}$ & $\mathbf{3 0}$ \\
\hline
\end{tabular}

Table 4. Distribution of labels

\subsection{Application of the algorithm}

A summary of the results is given in Table 5. As it is shown in Table 5, the maximal value of overall homogeneity degree corresponds to partition $k=3$ with $\alpha=0.8$ and $\beta=0.9\left(D P_{0.8,0.9}\left(P_{3}\right)=0.91\right)$

The justification of this result follows. First, note that for partition $P_{3}$, objects of the sets $E_{3}$ and $E_{4}$ are in the same class, which is $C_{2}$. Accordingly, all objects described by label $l_{4}$ are in class $C_{2}$. This leads to $M D_{\alpha}\left(l_{4}, C_{2}\right)=$ 1. Moreover, class $C_{2}$ contains the majority of objects described by label $l_{8}$. For $P_{4}$, in turn, the values of $M D_{\alpha}\left(l_{4}, C_{2}\right)$ and $M D_{\alpha}\left(l_{4}, C_{3}\right)$ are less than 1 ; and the values of $M D_{\alpha}\left(l_{8}, C_{2}\right)$ and $M D_{\alpha}\left(l_{8}, C_{3}\right)$ will decrease. Consequently, $D P_{\alpha, \beta}\left(P_{4}\right)<D P_{\alpha, \beta}\left(P_{3}\right)$. The same remark holds by comparing partition $P_{3}$ to partitions $P_{2}$, and $P_{5}$.

\begin{tabular}{|c|c|c|c|c|}
\hline$k$ & $\alpha_{\max }$ & $\beta_{\max }$ & Partition & $D P_{\alpha, \beta}$ \\
\hline 2 & 0.8 & 0.9 & $\begin{array}{l}C_{1}=\left\{50 \text { of } E_{1}, 40 \text { of } E_{2}\right\} \\
C_{2}=\left\{40 \text { of } E_{3}, 70 \text { of } E_{4}, 30 \text { of } E_{5}\right\}\end{array}$ & 0.88 \\
\hline 3 & 0.8 & 0.9 & $\begin{array}{l}C_{1}=\left\{50 \text { of } E_{1}, 40 \text { of } E_{2}\right\} \\
C_{2}=\left\{40 \text { of } E_{3}, 70 \text { of } E_{4}\right\} \\
C_{3}=\left\{30 \text { of } E_{5}\right\}\end{array}$ & 0.91 \\
\hline 4 & 0.8 & 0.8 & $\begin{array}{l}C_{1}=\left\{50 \text { of } E_{1}, 40 \text { of } E_{2}\right\} \\
C_{2}=\left\{40 \text { of } E_{3}\right\} \\
C_{3}=\left\{70 \text { of } E_{4}\right\} \\
C_{4}=\left\{30 \text { of } E_{5}\right\}\end{array}$ & 0.87 \\
\hline 5 & 0.4 & 0.8 & $\begin{array}{l}C_{1}=\left\{50 \text { of } E_{1}\right\} \\
C_{2}=\left\{40 \text { of } E_{2}\right\} \\
C_{3}=\left\{40 \text { of } E_{3}\right\} \\
C_{4}=\left\{70 \text { of } E_{4}\right\} \\
C_{5}=\left\{30 \text { of } E_{5}\right\}\end{array}$ & 0.79 \\
\hline
\end{tabular}

Table 5. Results of the application of Algorithm 1

\subsection{Discussion}

This section first explains the role of the parameters $\alpha$ and $\beta$ and their utility in explaining the behavior of datasets. A practical example illustrating this fact is then provided.

\subsubsection{Role of $\alpha$ and $\beta$}

First, we note that to obtain coherent results, the values of $\alpha$ and $\beta$ should be greater or equal to 0.5 . This corresponds to the majority rule. Indeed, according to Proposition 2, if $\alpha \geq 0.5$ and if $M D_{\alpha}\left(l_{j}, C_{i}\right)>0$, then class $C_{i}$ will contain more than $50 \%$ of objects having the label $l_{i}$. Moreover the fact that $\beta$ have to be greater than 0.5 assures that the number of labels $l_{j}$ of which $M D_{\alpha}\left(l_{j}, C_{i}\right)>0$ represents a proportion greater than $50 \%$ in the class. For instance, for partition $P_{3}$ in Table 5, we have:

- For $C_{1}$ : 
- $M D_{0.8}\left(l_{1}, C_{1}\right)=1$,

- $M D_{0.8}\left(l_{3}, C_{1}\right)=0.8$,

- $M D_{0.8}\left(l_{5}, C_{1}\right)=1$,

- $M D_{0.8}\left(l_{9}, C_{1}\right)=0.93$ and

- $H C_{0.8,0.9}\left(C_{1}\right) \geq 0.9$.

Then, class $C_{1}$ is described by labels $l_{1}, l_{3}, l_{5}$ and $l_{9}$.

- For $C_{2}$ :

- $M D_{0.8}\left(l_{2}, C_{2}\right)=1$,

- $M D_{0.8}\left(l_{4}, C_{2}\right)=1$,

- $M D_{0.8}\left(l_{6}, C_{2}\right)=1$,

- $M D_{0.8}\left(l_{7}, C_{2}\right)=0.89$,

- $M D_{0.8}\left(l_{8}, C_{2}\right)=0.8$ and

- $H C_{0.8,0.9}\left(C_{2}\right) \geq 0.9$.

Then, $C_{2}$ is described by labels $l_{2}, l_{4}, l_{6}, l_{7}$ and $l_{8}$.

- For $C_{3}$ :

- $M D_{0.8}\left(l_{10}, C_{3}\right)=1$, and

- $H C_{0.8,0.9}\left(C_{3}\right) \geq 0.9$.

Then, $C_{3}$ is described by label $l_{10}$.

This type of result can not be obtained with methods described in Section 2 since te use of a distance measure that handles mixed attributs does not grantee that the obtained classes are described by distinct labels.

Results such as ones presented above allow, in a second stage, to make decisions such as:

- Forecasting: the fact that each class is described with distinct labels permits to conclude that each object having a label $l_{j}$ must be included in a class for which label $l_{j}$ is representative. This permits to predict the behavior of any object.

- Detection of outlier: a new object $O_{r}$ described by a label $l_{j}$ is included in the nearest class $C_{i}$ in terms of numerical attributes. If the label $l_{j}$ is not representative of class $C_{i}$, then object $O_{r}$ is an outlier.

\subsubsection{Road traffic application}

Consider that a road traffic datasets representing the number of vehicles passing in a point $x$ of a road network each hour during one day. Each object is then described by 24 numerical attributes $\left\{A_{1}, A_{2}, \ldots, A_{24}\right\}: A_{1}$ is the number of vehicles on 00h00, $A_{2}$ is the number of vehicles on $01 \mathrm{~h} 00$, and so on. The objects are also described by 2 categorical attributes $A_{25}$ and $A_{26}$ that represent respectively working day and holiday with $\operatorname{DOM}\left(A_{25}=\{Y, N\}\right.$ and $\operatorname{DOM}\left(A_{26}\right)=\{Y, N\}$.

By applying one of numerical attributes-based clustering methods, the proposed approach assures that the objects in the same class are more similar than objects within different classes according to only numerical attributes. After that, the identification of the partition that maximizes the overall homogeneity degree permits to detect factors that influence the behaviors of datasets. For example, obtaining a class $C_{1}$ described by the label $N N$ and $N Y$ ( $\alpha$ and $\beta$ should be greater or equal to 0.5 ) indicates that the majority of the objects described by label $N N$ or label $N Y$ are in the same class. We can next conclude that the traffic during weekend is similar to the traffic during the holiday and that any new object whose values are recorded in a holiday or a weekend will have the same behavior as the representative labels of class $C_{1}$. Moreover, we can detect the outliers as mentioned above and we can explain some facts like accidents or traffic lights problems.

\section{Conclusion}

In this paper, we first introduced the concept of homogeneity degree. This concept permits to measure the level to which a class is homogenous in respect to categorical attributes. Then, we proposed an extended mixed-attributes clustering algorithm based on the notion of homogeneity degree. Currently, the extended algorithm requires the use of a conventional partitional clustering method. However, we intend to explore the possibility to extend the algorithm to apply with other type of clustering methods such as hierarchical or density-based ones.

\section{References}

[1] A. Ahmed and D. Lipika. A k-mean clustering algorithm for mixed numeric and categorical data. Data and Knowledge Engineering, 63(2):503-527, 2007.

[2] H. Chung-Chian, C. Chin-Long, and S. Yu-Wei. Hierarchical clustering of mixed data based on distance hierarchy. Information Sciences, 177(20):4474-4492, 2007.

[3] M. Ester, H.-P. Kriegel, J. Sander, and X. Xu. A densitybased algorithm for discovering clusters in large spatial databases with noise. In Proc. of 2nd Int. Conf. on Knowledge Discovery and Data Mining (KDD), pages 226-231, Portland, Oregon, August 1996.

[4] S. Guha, R. Rastogi, and K. Shim. CURE: An efficient clustering algorithm for large databases. In Proc. of ACM SIGMOD Int. Conf. on Management of Data, pages 73-84, Seatle, USA, June 1998.

[5] M. Halkidi, Y. Batistakis, and M. Vazirgiannis. On clustering validation techniques. Journal of Intelligent Information Systems, 17(2):107-145, 2001. 
[6] Z. He, X. Xu, and S. Deng. Scalable algorithms for clustering mixed type attributes in large datasets. International Journal of Intelligent Systems, 20(10):1077-1089, 2005.

[7] Z. Huang. A fast clustering algorithm to cluster very large categorical data sets in data mining. In Research Issues on Data Mining and Knowledge Discovery, 1997.

[8] Z. Huang. Extensions to the k-means algorithm for clustering large data sets with categorical values. Data Mining and Knowledge Discovery, 2(3):283-304, 1998.

[9] A.-K. Jain, M. Murty, and P.-J. Flynn. Data clustering: A review. ACM Computing Surveys, 31(3):264-323, 1999.

[10] L. Kaufman and P. Rousseeuw. Finding groups in data: An introduction to cluster analysis. John Wiley \& Sons, 1990.

[11] C. Li and G. Biswas. Unsupervised clustering with mixed numeric and nominal data- a new similarity based agglomerative system. In Proc. of the First Asian Pacific Conference on Knowledge Discovery from Databases, pages 35-61, Singapore, 1997.

[12] J. Mcqueen. some methods for classification and analysis of multivariate observations. In 5th Berkeley Symp. on Math. Statistics and Probability, pages 281-298, Berkley, CA: University of California Press, 1967.

[13] R.-T. Ng and J. Han. Efficient and effective clustering methods for spatial data mining. In 20th Int. Conf. on Very Large DataBases (VLDB), pages 144-155, Santiago, Chile, September 1994.

[14] H. Ralambondrainy. A conceptual version of the k-means algorithm. Pattern Recognition Letters, 16(11):1147-1157, 1995.

[15] T. Zhang, R. Ramakrishnan, and M. Livny. BIRCH: An efficient data clustering method for very large databases. In Proc. of ACM SIGMOD Int. Conf. on Management of data, Montreal, Canada, June 1996. 